\title{
EL MALTRATO A LAS PERSONAS MAYORES DESDE EL PARADIGMA DE LA VIOLENCIA
}

\author{
THE ABUSE OF ELDERLY PEOPLE FROM THE PARADIGM OF THE \\ VIOLENCE
}

\author{
José Luis Paniza Prados \\ Juan Carlos Ortigosa Perochena \\ Universidad de Granada. España/Spain \\ jpaniza@ugr.es \\ jcopi@,telefonica.net
}

Recibido/Received: 08/04/2015

Modificado/Modified: 30/09/2015

Aceptado/Accepted: 04/10/2015

\section{RESUMEN}

El presente artículo se suma al debate metodológico y sobre todo teórico utilizado para el estudio de la violencia hacia el mayor. Para ello, nos proponemos conceptualizar y acotar correctamente un término como el de violencia, tarea que consideramos imprescindible a la hora de definir la violencia que se ejerce sobre la persona mayor, determinando cuales son los aspectos distintivos del acto violento. También, realizaremos un breve repaso de las distintas teorías de la violencia que son relevantes desde el punto de vista sociológico y que permiten explicar el fenómeno de los malos tratos a los mayores. Finalmente analizaremos que definición y que teorías se adecuan más al estudio de la violencia ejercida contra el mayor.

\section{PALABRAS CLAVE}

Sociología, violencia, maltrato, mayores.

\section{SUMARIO}

1. Introducción. 2. Definición de violencia. 3. Algunas teorías sobre la violencia. 3.1. Teoría utilitarista. 3.2. Las teorías culturalistas. 3.3. Teoría de la frustración-agresión. 3.4. Teoría del etiquetaje. 3.5. Teoría de la violencia creativamente orientada. 3.6. La teoría de las emociones y la violencia. 3.7. Teoría ecológica. 3.8. Teoría de la violencia en red. De lo micro a la macro. 4. Conclusión. 5. Bibliografía.

\begin{abstract}
This paper adds to the methodological and theoretical debates used in the study of violence exercised towards the elderly. Thus, we propose to conceptualize and refine properly the term "violence", a task that we consider crucial when defining and characterizing the different aspects of violent acts against elderly people. Additionally, we conduct a brief revision of the different theories regarding violence that are relevant within the sociological context and allow us to explain the phenomenon of the abuse of the elderly. Finally, we analyze which definitions and theories are the most appropriate for the study of the violence against the elderly.
\end{abstract}

\section{KEYWORDS}

Sociology, violence, abuse, elderly. 


\section{CONTENTS}

1. Introduction. 2. Definitions of violence. 3. Different theories about violence. 3.1. Utilitarian theory. 3.2. Cultures theories. 3.3 Frustration-aggression theory. 3.4. Labeling theory. 3.5. Theory of the creative-oriented violence. 3.6. Theory of emotions and violence. 3.7. Ecological theory. 3.8. Network violence theory. 4. Conclusion. 5. Bibliography.

\section{INTRODUCCIÓN}

Los malos tratos a mayores constituyen una realidad dramática que en las últimas décadas está recibiendo una mayor atención por parte de la sociedad, los medios de comunicación y los investigadores sociales. Los primeros estudios sobre la temática se desarrollan en Estados Unidos a finales de los setenta, unas décadas después, con la Declaración de Toronto (2002), los malos tratos a mayores adquieren una dimensión internacional y se empieza a considerar un problema social en occidente.

A nivel internacional, la Organización Mundial de la Salud, indica que el porcentaje de maltrato al mayor (de la prevalencia de los malos tratos) varía entre el 1 y el 35 por ciento, dependiendo del proceso de investigación realizado, en lo relativo a la definición y conceptualización del maltrato, de la metodología empleada y de la muestra utilizada; de hecho, algunos expertos creen que el maltrato a mayores está infravalorado hasta en un 80 por ciento (WHO, 2008:1).

En España, las investigaciones realizadas hasta la fecha, arrojan tasas de malos tratos bajas o muy bajas. La estimación de la incidencia estaría alrededor del 5 por ciento, si atendemos a los opiniones emitidas por los cuidadores de las personas mayores; sin embargo, las victimas sólo reconocen una tasa de maltrato cercana al 0,8 por ciento (Iborra, 2008). Esto es algo que se repite a lo largo de los estudios consultados, parece que hay mayor sinceridad en los causantes de los malos tratos que en los propios afectados. Lo que nos lleva a pensar que existe una resistencia insuperable por parte del mayor a confesar los malos tratos a pesar del anonimato que se les declara.

La discrepancia y divergencia de los datos obtenidos en las diferentes investigaciones nos muestran que algo falla de manera generalizada en las investigaciones sobre el abuso o violencia contra las personas mayores; en los 28 seminarios ofrecidos por UDP (Unión Democrática de Pensionistas y Jubilados de España) desde abril de 2003 se ha puesto de manifiesto por especialistas de todas las disciplinas la necesidad de emplear metodologías comunes de investigación para el estudio, prevención y tratamiento de este problema social.

El presente artículo se suma al debate metodológico y sobre todo teórico utilizado para el estudio de la violencia hacia el mayor. Para ello, nos proponemos en primer lugar conceptualizar y acotar correctamente un término como el de violencia, tarea que consideramos imprescindible a la hora de definir la violencia que se ejerce sobre la persona mayor, determinado cuales son los aspectos distintivos del acto violento.

También, realizaremos un breve repaso de las distintas teorías de la violencia que son relevantes desde el punto de vista sociológico y que permiten explicar el fenómeno de los malos tratos a los mayores. Finalmente analizaremos que definición y que teorías se adecuan más al estudio de la violencia ejercida contra el mayor. 


\section{DEFINICIÓN DE VIOLENCIA.}

La percepción social que se tiene de la violencia cambia con el paso del tiempo y por supuesto en función del espacio geográfico y social. Aquello que se considera como un hecho violento en un momento histórico deja de serlo en otro; del mismo modo, lo que considera violento un grupo que ha sido derrotado es considerado heroico por aquél que ha triunfado. Cada cultura, cada sociedad, ha definido en cada momento, lo que tolera, acepta o rechaza, incluso aunque no se corresponda con las categorías fijadas en ese instante por la ley, o el derecho; de tal manera que la forma que adquiere la legitimación de la violencia, nos revela lo que piensa la sociedad en un momento histórico y en un lugar determinado (Wieviorka, 2005:75).

La Organización Mundial de la Salud (OMS), en el capítulo 1 de su Informe Mundial sobre la Violencia y la Salud referido a la violencia en general, define la violencia como "el uso intencional de la fuerza o el poder físico, de hecho o como amenaza, contra uno mismo, otra persona, o un grupo o una comunidad, que cause o tenga muchas probabilidades de causar, lesiones, muerte, daños psicológicos, trastornos del desarrollo o privaciones" (OMS, 2002:5). Como se ve, trata de abarcar distintos aspectos de la violencia tanto en su concepción como en sus consecuencias. De hecho, la OMS indica que "muchas formas de violencia contra las mujeres, niños y mayores, por ejemplo, pueden dar como resultado problemas físicos, psicológicos y sociales que no desembocan necesariamente en lesión, incapacidad o muerte" (OMS, 2002:5).

La OMS define también la violencia teniendo en cuenta su relación con la salud o el bienestar de las personas y con independencia del hecho cultural. "Ciertos comportamientos como golpear al cónyuge- pueden ser considerados por algunas personas como prácticas culturales admisibles, pero se consideran actos de violencia con efectos importantes para la salud de la persona" (OMS, 2002:6).

El sociólogo e historiador Charles Tilly nos destaca el factor oportunidad en su definición de la violencia; significando como características diferenciales de la misma, la activación de las líneas divisorias disponibles nosotros-ellos; la respuesta a una represión debilitada, desviada o fallida; los espirales de señales que indican que determinadas prácticas generalmente arriesgadas resultan ahora posibles y efectivas y que, por lo tanto, hacen variar la disposición de los participantes a asumir los riesgos de los que se trate; y, por último, la represalia selectiva por daños experimentados con anterioridad (2007:133). Como se observa, son aspectos todos ellos presentes en un momento $u$ otro de una sociedad determinada.

Wieviorka opta por no ofrecer una definición de la violencia, ya que la considera difícil de definir porque en una definición de carácter objetivo como la que hemos citado de la OMS, se echa en falta la subjetividad del autor, de la víctima o del observador. El "nuevo" sujeto de la violencia, la víctima, establece para Wieviorka (2005) un nuevo paradigma a partir de los años 60 o 70. En efecto, los razonamientos clásicos son para él a la vez útiles, pero insuficientes y desemboca en una atención central en la subjetividad de los actores. También Joas (2005) destaca el punto de vista subjetivo, y pone el acento en la creatividad del autor del hecho violento. El aspecto individual, singular, del acto violento en los malos tratos a mayores aparece más destacable por sus propias características, pero no debemos olvidar su carácter social, al estar enraizado en determinadas actitudes o conductas y valores sociales.

El papel destacado que se dedica a la subjetividad hace que sea más sencillo situar la violencia no sólo en su aspecto colectivo, sino también individual e interrelacionar los 
aspectos individuales y sociales de la violencia contra los mayores. El Estado tolera poco la violencia sufrida por las mujeres, los niños o las personas mayores. En la actualidad permite menos que la vida privada o familiar, así como también algunas instituciones, incluidas la escuela y las iglesias, se constituyan en enclaves cerrados a su control. Las instituciones se vuelven más sensibles a los problemas de las personas singulares, es decir, las instituciones miran hacia los sujetos individuales dejando de estar sacralizadas (Wieviorka, 2005:75, 89). Si bien, hay que poner de relieve que en el caso de los malos tratos a los mayores, el camino recorrido es muy corto y hay mucho terreno por delante hasta que este problema sea considerado un problema social.

Ahora bien, la violencia precisa, además del punto de vista psicológico, de un enfoque sociológico, como problema social es necesario examinar las conductas y valores sociales. Luis Rojas Marcos, aunque es psiquiatra, está vivamente interesado en las relaciones sociales, en general, y en la cuestión de la violencia, en particular. Su posición sociológica queda definida en su obra sobre la violencia desde el comienzo: "la agresión maligna no es instintiva sino que se adquiere, se aprende. Las semillas de la violencia se siembran en los primeros años de vida, se cultivan y desarrollan durante la infancia y comienzan a dar sus frutos malignos en la adolescencia. Estas simientes se nutren y crecen estimuladas por los ingredientes del medio hasta llegar a formar una parte inseparable del carácter del adulto. Los seres humanos heredamos rasgos genéticos que influyen en nuestro carácter. Pero nuestros complejos comportamientos, desde el sadismo al altruismo, son el producto de un largo proceso evolutivo condicionado por las fuerzas sociales y la cultura." (Rojas Marcos, 2008: 14). Esta magnífica exposición plantea la génesis y, en definitiva, la definición de la violencia, desde un punto de vista social y cultural.

Consideramos que es imprescindible mantener la necesaria dialéctica entre el concepto de violencia como universal abstracto y totalmente objetivado y la que se produce en una persona o un colectivo concreto y en un momento histórico determinado. Si nos limitamos al primer nivel perdemos la perspectiva singular pero si únicamente nos quedamos en el segundo llegaríamos a un completo relativismo que no se fijaría más que en lo particular y haría imposible el análisis. Mantener la vista en los dos aspectos con las variaciones del momento es lo que puede permitir un análisis más fructífero.

A pesar de que compartimos con Wieviorka que dar una definición de la violencia es acotar el ámbito de ésta y, por tanto, limitarla, no queremos dejar pasar la ocasión de poner de manifiesto las notas más destacables que, en nuestra opinión, caracterizan los actos violentos y dar a continuación una definición propia. Destacamos los siguientes aspectos de la violencia:

* El acto puede ser intencionado o no. Hemos hecho hincapié en que creemos que es indiferente desde el punto de vista del agredido. Por lo tanto cualquier acto violento lo es independientemente de la intencionalidad del victimario.

* La pueden ejercer no sólo los individuos o grupos sino también instituciones estatales, sociales o religiosas, y otros grupos de poder como los económicos. La ejerce especialmente el Estado cuando incumple las normas y leyes que ha dictaminado respecto del bienestar, atención, asistencia social y económica de los mayores.

* El acto violento se valora desde el punto de vista del daño a la víctima, sea ésta un individuo, grupo o colectividad, y no del victimario, y es independiente del momento histórico, lugar geográfico o de la cultura y religión de los actuantes.

* Además del daño o la coacción física, consideramos violentas aquellas actuaciones que causen o tengan probabilidades de causar privaciones que conlleven la pérdida de la dignidad personal, el bienestar económico básico, la separación no querida del entorno 
social más cercano y otras de signo parecido.

* Aquellos actos que puedan causar lesiones, muerte, daños psicológicos y trastornos del desarrollo.

Una vez determinados los aspectos distintivos del acto violento, definimos violencia como: todo tipo de actuación llevada a cabo por individuos, grupos o cualquier tipo de institución o grupo de poder, que provoque un daño físico, moral o de privación y conlleve lesiones, muerte o atente contra la dignidad personal o produzca la separación no querida del entorno personal más cercano.

Hacemos hincapié no solamente en la fuerza o el poder físico que la violencia involucra, pues en este momento histórico más que la agresión física directa, se utilizan con frecuencia los medios institucionales del Estado o de otros grupos de poder sociales, económicos o religiosos para dañar gravemente a personas o colectivos tanto por acción como por omisión.

\section{ALGUNAS TEORÍAS SOBRE LA VIOLENCIA}

En el presente epígrafe realizamos un breve repaso histórico de las distintas teorías de la violencia que son relevantes desde el punto de vista sociológico y que permiten explicar el fenómeno de los malos tratos a los mayores. En concreto nos centraremos en la teoría utilitarista, la teoría cultural, la teoría de la frustración - agresión, la teoría del etiquetaje, la teoría de la violencia creativamente orientada, la teoría de las emociones, la teoría ecológica y la teoría de la violencia en red. Sin embargo, no vamos a tener en cuenta a Freud con la teoría de los instintos innatos, ni a etólogos como Lorenz, que si bien han supuesto una importante aportación, actualmente están ampliamente superados por los descubrimientos y aportaciones de la neurociencia y la sociobiología (Homans, 1990).

\subsection{Teoría utilitarista}

En la teoría utilitarista, la violencia constituye un útil, un recurso, un instrumento, utilizado de forma racional por los actores que calculan, elaboran estrategias y hacen de ellas un medio al servicio de sus fines (Wieviorka, 2005:161). Joas (2005) destaca el utilitarismo como una de las dos teorías dominantes para explicar la violencia. Es una violencia de tipo socioestructural, ya que destaca en el origen los círculos de intereses como causantes de la misma.

Hobbes es el primer teórico que aplica el principio utilitarista para explicar la violencia instrumental: "el hombre es un lobo para el hombre" y que, por tanto, es capaz de todo con tal de conseguir sus fines. Dicha afirmación, tiene plena vigencia para explicar muchas de las causas de la violencia pero, naturalmente, no explica todas y el papel de la víctima no se pone de manifiesto o queda desdibujado.

\subsection{Las teorías culturalistas}

En este tipo de teorías, la cultura, constituye la raíz de la violencia, se pone el acento en los valores, o más concretamente en la pérdida de los valores. Las teorías culturalistas pueden clasificarse en función del peso que tienen en ellas diferentes aspectos de la misma en: teorías sobre la personalidad autoritaria, teorías conductistas y teorías ideológicas.

La teoría sobre la personalidad autoritaria "entiende que es posible asociar los trazos de personalidades autoritarias y antidemocráticas a la cultura en la que estos trazos se han 
formado o reproducidos en la infancia" (Adorno, 1965). El acento se pone en la personalidad y, por tanto, en la educación y en sus consecuencias en las personas adultas. Pero también la violencia puede venir de tipos considerados como ordinarios. Es un análisis que nos sigue pareciendo explicativo de ciertas conductas violentas en el ámbito familiar y, desde luego, en la violencia contra los mayores tanto por parte de familiares o cuidadores como en ámbitos institucionales. Esta visión nos sigue sirviendo de ayuda para reflexionar sobre la pedagogía y su importancia en la transmisión de valores. Algunas de las polémicas más agudas que atraviesan nuestra sociedad en este momento son sobre el tipo de educación que debemos adoptar y sus consecuencias en la adquisición de valores para la madurez.

No queremos dejar de citar entre las teorías sobre la violencia el conductismo, que ha sido y es todavía en sus diferentes formas de gran fecundidad en los estudios sobre la agresión en psicología y ha desarrollado las teorías del aprendizaje social. Para el conductismo, el elemento constitutivo de los comportamientos proviene de la asociación de una determinada respuesta a un estímulo concreto. Ciertos estímulos provocan cierta clase de comportamientos agresivos, los cuales se hacen permanentes por el refuerzo que se ha producido de sus ejecuciones tras cada una de las apariciones del estímulo. Cuando el comportamiento agresivo de una persona es reforzado entonces lo puede aprender. La aportación de Bandura ha sido fecunda en la comprensión del comportamiento agresivo, sobre todo porque ha permitido distinguir analíticamente el aprendizaje de un comportamiento y su ejecución. Bandura nos dice que estos comportamientos se aprenden pero su aprendizaje dista mucho de su ejecución.

La ideología ha sido utilizada como generadora de violencia. La ideología constituye la justificación de los hechos consumados y el discurso se explica sin pasar por la prueba de lo real. Esto ocurre mucho más a menudo de lo que nos parece razonable: la violencia que se justifica en las ideas, pero como principio, sin necesidad de contrastación (Arendt, 2014). Pero también de victimarios de pareja o de mayores porque se basan en su propia superioridad por definición, como axioma que no necesita demostración. Supone la desvalorización del otro al que, por tanto, se puede destruir. También la sociedad patriarcal dominante está en el origen de muchas de las conductas agresivas y violentas.

Del mismo modo, la banalización de todas las conductas como iguales y sin ningún valor diferencial, ni moral ni social, puede estar en el origen de algunas conductas violentas y agresivas. Dicha conducta banalizadora se caracteriza también por la falta total de empatía con los demás y, por supuesto, con la víctima.

Sabemos que el análisis de la violencia basada en la cultura puede explicar algunos tipos, pero al igual que en el caso del utilitarismo no lo explica todo y además, el mismo concepto de cultura no es unívoco y, por tanto, puede añadir confusión al análisis.

\subsection{Teoría de la frustración-agresión}

La teoría de la frustración-agresión supone un avance en cuanto a la influencia ambiental, ya que nos dice esencialmente que la agresión es un comportamiento resultante de una pulsión interna pero esta pulsión depende de un elemento externo: la frustración para que se desencadene la agresión (Doménech e Íñiguez, 2002).

La crítica de esta teoría es que la agresión es una de las consecuencias de la frustración y que la frustración acumulada no es la única causa de la agresión (Hewstone, 1994). En algunas ocasiones, la reacción violenta acontece cuando de una situación de abundancia se pasa a otra de carencia sin solución de continuidad. Supone una reacción a la teoría funcionalista y tuvo bastante aceptación y difusión a principios de los setenta. Sin 
embargo, la teoría de la frustración-agresión, cuando se analizan los hechos concretos, da lugar a muchos contraejemplos que la refutan.

\subsection{Teoría del etiquetaje}

La desviación en una sociedad depende de lo que ésta considere como desviado, de ahí la denominación de teoría del etiquetaje. La consideración de lo que se considera violencia y los diferentes tipos cambian a lo largo del tiempo en un sentido diacrónico y en distintos lugares, en sentido sincrónico. Recordamos que mientras una conducta, en nuestro caso el maltrato a mayores, no se etiquete de forma fehaciente como desviada y como problema social, este problema no saldrá a la luz pública. Para Giddens (2010:163), "las etiquetas utilizadas para crear categorías de desviación expresan, por tanto, la estructura de poder de la sociedad", y concluye con que "la teoría del etiquetaje es quizá el enfoque más amplio para comprender aspectos del delito y de la conducta desviada".

Esta teoría nos parece muy interesante para analizar las condenas o la justificación que se hacen de muchas conductas según el momento histórico que se vive y el lugar geográfico que se ocupa. A veces lo que se pueden observar como conductas violentas son autodefensas ante situaciones de injusticia donde los cauces democráticos se encuentran cerrados. Por otro lado, si como hemos dicho más arriba, conductas que son claramente dañinas para grupos en situación social de debilidad u opacidad no se vuelven visibles (no se etiquetan) no tendrán ni la prevención ni la reparación debida.

La psicología social contemporánea ubica la agresión en un contexto de interacción interpersonal e intergrupal. Una nota destacada es que para considerarse un comportamiento como agresivo debe ser antinormativo. En relación con este asunto, "la conducta agresiva implica una forma especial de influencia social: un individuo obliga a otro a hacer algo que no habría hecho sin esa coerción. La agresión, por tanto, consiste en la aplicación del poder coercitivo, Ya sea en forma de amenaza o castigo." (Hewstone, 1990:277).

\subsection{Teoría de la violencia creativamente orientada}

Para Joas (2000/2005:253, 255) existen dos modelos que explican la violencia: uno de tipo utilitarista y otro basado en valores culturales. No obstante, nos dice que aunque ambas teorías no son descartables a priori "se quedan relativamente mudas cuando se trata de saber en qué momento se produjo la irrupción de la violencia, así como la dinámica interna del hecho violento o la extensión del mismo". Por ello, añade a estos dos modelos de explicación de la violencia un tercero, superior a los otros dos, que es el modelo creativamente orientado. Este modelo aborda dos cuestiones que se quedan sin resolver en los otros dos. Por una parte, la de cómo emplear las normas y los valores en situaciones de acción concretas $\mathrm{y}$, por otra, la de la cuestión acerca del surgimiento de los valores orientados a la acción propiamente dicha.

La acción violenta, individual, singular, tiene en sí misma un aspecto de creatividad ya que no se limita a reproducir las condiciones, sean de tipo utilitarista o cultural, sino que añade una parte propia perteneciente al individuo. Sin olvidar que la violencia individual, está siempre en una sociedad concreta que puede justificar, silenciar o ignorar un tipo de acciones violentas. Destaca el aspecto individual de la violencia y, por tanto, debemos analizar, el carácter intencional de la acción humana, la corporeidad específica y la socialidad originaria de la capacidad humana para la acción. (Joas, 2000/2005:256). Este autor rinde tributo en el origen de su modelo a las teorías simbólico-interaccionistas. 


\subsection{La teoría de las emociones y la violencia}

Queremos hacer una breve mención de la teoría de las emociones. Para nosotros, la neurociencia tiene cada vez mayor importancia en la explicación de toda la actividad humana y debe ser integrada, como un elemento valioso, en las explicaciones sociológicas.

En este sentido, aunque la mayor parte de los psicólogos cree que hay ciertas emociones "básicas" que se encuentran en todos los humanos, es claro que las percepciones de los individuos, de lo que es relevante para su "bienestar o aflicción", producirá muchas clases diferentes de emociones en situaciones similares. Esta variedad de reacciones hace que no se puedan encorsetar y reducir las prácticas concretas a un número limitado de tipos. Pero esto, en nuestra opinión, no significa, como hemos apuntado antes, que no podamos analizar y clasificar los malos tratos según un acervo casi común. Damasio (1999) sugiere que nuestro cerebro reacciona inconscientemente a los estímulos, pero esto no niega las bases de la teoría del conocimiento por cuanto la respuesta del cerebro implica conocimiento y evaluación.

La capacidad física y mental para tener emociones es universal, pero cómo estas mismas emociones son provocadas, sentidas y expresadas, depende de las normas culturales así como de las tendencias propias de cada individuo. No se debe, no obstante, negar el sustrato biológico de las emociones, ya que es claro que están embebidas en el cuerpo y el cerebro. Al mismo tiempo, una historia de las emociones debe de problematizar los sentimientos del pasado, abordando sus características distintivas. Las emociones, o mejor dicho, la importancia de los cambios que sufren las emociones según el periodo histórico y el lugar, hacen que "al final, los problemas y métodos de la historia de las emociones deberían volverse el terreno de la historia en general.” (Rosenwein, 2010:24).

\subsection{Teoría ecológica}

En su informe mundial sobre la violencia y la salud, la OMS aplica el modelo ecológico como base para la explicación de la violencia y, específicamente, la violencia contra los mayores. Este modelo se utilizó primero para explicar el maltrato de niños; después la violencia de los jóvenes; y más recientemente la violencia de las parejas y también la del maltrato a mayores.

El modelo explora la relación entre los factores individuales y del contexto considerando la violencia como el producto de los diversos niveles de influencia sobre el comportamiento: (OMS, 2002:13).

* El primer nivel del modelo ecológico busca identificar los factores históricos, biológicos y personales que influyen en el comportamiento de una persona.

* El segundo nivel se refiere a la influencia de las relaciones sociales próximas en el aumento del riesgo de que alguien sea víctima o perpetrador de violencia.

* El tercer nivel examina el contexto comunitario en el que se desarrollan las relaciones de la persona como escuelas, lugares de trabajo y vecindarios.

* El cuarto y último nivel examina los factores sociales más amplios que influyen en el grado de la violencia.

La virtud de este modelo es que enfoca las múltiples causas de la violencia y la interacción de los factores de riesgo que operan dentro de la familia y la comunidad, así como los contextos sociales, culturales y económicos. Trata de integrar, desde lo más cercano a lo más general, todas las posibles influencias que se pueden dar para favorecer los factores de riesgo de los malos tratos. 


\subsection{Teoría de la violencia en red. De lo micro a lo macro social}

Esta visión de la violencia quiere establecer un enlace entre los niveles micro y macro social, entre lo interpersonal y lo global, y por lo tanto, una red de la violencia en que lo predominante a nivel general (o macro social) se transfiere y se traspone también al nivel micro social que incluye las relaciones intrafamiliares y sociales más cercanas.

Un ejemplo que ayuda a entender esta teoría es la cultura patriarcal de nuestra sociedad. Diferentes estudios han puesto de manifiesto que el dominio de lo masculino sobre lo femenino crea violencia contra las mujeres y los niños en un nivel micro. La ideología sexista, perpetúa la violencia sistémica desde la casa hasta el orden político global. En esta misma línea, otros investigadores han demostrado la naturaleza de género de la violencia de lo interpersonal a lo global, y su relación con la socialización según sexos y la división sexista de los recursos en todos los niveles de la sociedad (Turpin y Kurtz, 1997:10).

En esta misma línea de pensamiento, Robert Elias (1997) hace hincapié en el mantenimiento de la sociedad patriarcal como causa principal de la violencia. Nos dice que la violencia familiar ha crecido hasta proporciones epidémicas y este crecimiento no solo es el resultado de la sociedad patriarcal sino que también le proporciona un medio principal para reforzarla. Destacamos de nuevo que se refiere al maltrato de niños y mujeres y no dice nada de la violencia contra los mayores pero se puede aplicar perfectamente a este tipo de maltrato.

Otro ejemplo de esta conexión entre los niveles micro y macro es el que distingue entre violencia pública o privada; así, en un mundo en que los derechos humanos fueran verdaderamente valorados, la crueldad, la opresión y la discriminación, se verían como absurdos (Eirler, 1997:163). Precisamente la interrelación entre el nivel microsocial y el macrosocial de la violencia se da desde las relaciones patriarcales intrafamiliares e interfamiliares que crean el caldo de cultivo para su traslado hacia la violencia de la sociedad en la que están inmersos y controlan. O al revés, la sociedad controlada por el tribalismo, el patriarcalismo o la religión, traen como consecuencia con cierta frecuencia unas relaciones sociales e intrafamiliares violentas.

La privacidad, es decir, los derechos de una persona para tomar ciertas decisiones no es lo mismo que inmunizar ciertas decisiones familiares tomadas por aquellos que tienen el poder en la misma. Por tanto, la protección de los derechos personales no es sinónimo a la no interferencia en los actos en el interior de la familia. (Eirler, 1997:167). En ocasiones la privacidad del hogar es una excusa para ocultar los casos de violencia intrafamiliar.

Finalmente, consideramos que se hace necesario contraponer el modelo social de dominación con el de asociación. En el primero, se impone la superioridad frente a un inferior y la violencia se desata con facilidad; el único camino realista para romper los ciclos violentos -sean interpersonales o internacionales- es ayudar a las mujeres y varones, padres e hijos y mayores para que acaben con la aceptación de las relaciones de tipo violento. (Eiler, 1997:180)

Los actos de violencia que se dan en el nivel macrosocial tienen repercusión en el nivel microsocial ya que una situación de violencia generalizada o extendida se traslada a todos los ámbitos de la sociedad incluido el maltrato de los mayores, o el maltrato extendido a los mayores en las instituciones. Cuando se define una superioridad, los inferiores sufren indefectiblemente las consecuencias de esta definición. 


\section{CONCLUSIÓN}

Conceptualizar y acotar correctamente un término como el de violencia es una tarea imprescindible a la hora de definir la violencia que se ejerce sobre la persona mayor, por ello en el presente artículo hemos determinado cuales son los aspectos distintivos del acto violento, a saber: la intencionalidad, la autoría (institucional o particular) y el daño que se produce a la víctima ya sea de privación, psíquico o físico. Una vez definidos los aspectos del acto violento hemos visto pertinente definir la violencia como: todo tipo de actuación llevada a cabo por individuos, grupos o cualquier tipo de institución o grupo de poder, que provoque un daño físico, moral o de privación y conlleve lesiones, muerte o atente contra la dignidad personal o produzca la separación no querida del entorno personal más cercano.

En lo relativo a las diversas teorías que analizan la violencia pensamos que todas realizan aportaciones importantes al estudio de la violencia contra el mayor, aunque nos sentimos más próximos a una posición que aúna la teoría ecológica con la que, para nosotros, tiene un peso teórico más amplio que es la teoría de la violencia en red que establece el nexo entre los niveles macro y micro social.

Pensamos que el individuo es consciente siempre de alguna manera de sus actos, aunque nublado en cierto grado de su (mala) acción por el discurrir de su vida entre iguales que justifican o impulsan esta conducta. Los enmascara y justifica en la falta de valores y, todavía más, el mantenimiento oculto en la llamada esfera de lo privado que refuerza este enmascaramiento y la autojustificación. La manera de evitar este enmascaramiento es la existencia de una conciencia social del problema y la condena del mismo, para la implementación en la sociedad de ciertas actuaciones o condenas, más allá de la existencia de leyes condenatorias, hace falta una condena explícita de la mayoría de la sociedad de las conductas violentas.

Resulta evidente que viviremos más años, la longevidad que antes era una anomalía comienza a ser algo relativamente común en países como Japón, Francia y España, pero lo realmente importante para estas poblaciones son las condiciones físicas y sobre todo sociales en las que se va a producir esta extensión de la existencia.

La vejez no es una enfermedad, pero familiares e instituciones en numerosas ocasiones tratan al mayor como un problema, y hablamos del riesgo que supone para el sistema que cada vez más personas vivan más. Los mayores se convierten en una carga para el sistema y su cuidado y mantenimiento supone una carga social y económica para las generaciones venideras.

En este contexto resulta fácil que se desate la violencia contra el mayor, así, mientras factores biológicos y otros individuales explican algunas de las predisposiciones a la agresión, a menudo estos factores interaccionan con la familia, la comunidad, la cultura y otros factores externos para crear una situación en la que la violencia es fácil que ocurra, por lo tanto, si se cambiasen los factores externos también la violencia podría ser evitada (OMS, 2002).

Por consiguiente, las políticas de prevención son el primer remedio para evitar la violencia. Lo pone de manifiesto Rojas Marcos que destaca que el medio para disminuir la violencia no es el castigo sino la prevención y lo más adecuado es la aplicación de estrategias de salud pública (1995:212). También la OMS (2002) subraya la importancia de las medidas preventivas y la necesidad de que estas deben estar dentro del marco de un desarrollo de salud pública. La salud pública será, pues, un factor que favorece de forma destacable el bienestar general. Pero para que estas estrategias funcionen, primero hay que 
reconocer el problema, sacarlo a la luz y no mantenerlo en la esfera privada como algo sucio que no se debe mostrar. En lo que a los malos tratos contra los mayores se refiere, estamos todavía en la fase de la necesidad de darlo a conocer de forma extendida a la sociedad. Mientras tanto, hay medidas de prevención, de salud pública, que deben implementarse en forma de cribados y participación activa de todos los estamentos de atención a los mayores que puedan estar involucrados.

\section{BIBLIOGRAFÍA}

ADORNO, T. W. [et al.] (1965). La personalidad autoritaria. Buenos Aires: Proyección. ARENDT, H. (2014). Eichmann en Jerusalén. Barcelona: Debolsillo.

BARBERO, J. y MOYA, A. (comps.) (2006). Malos tratos a personas mayores: guía de actuación. Madrid: Imserso.

BAZO, M. T. (2003). "Violencia familiar contra las personas ancianas que sufren dependencia y enfermedad. Alternativas". Cuadernos de trabajo social, 11: 35-46, en: http://rua.ua.es/dspace/handle/ 10045/5272/browse?type=title\&submit_browse $=$ T\%C3\%ADtulo (consulta 28/7/2014)

BAZO, M.T. (2001). "Negligencia y malos tratos a las personas ancianas en España" Revista Española de Geriatría y Gerontología, 36 (1): 8-14, en http://zl.elsevier.es/es/revista/revista-espanola-geriatriagerontologia-124/sumario/vol-36-num-1-12001507 (consulta 28/7/2014).

BLOCK, M.R. y SINNOTT, J.D. (comp.) (1979). The battered elder syndrome: An exploratory study. College Park: University of Maryland Center on Aging.

BUŽGOVÁ, R. e IVANOVÁ, K (2009). "Elder Abuse and Mistreatment in Residential Settings", en Nurs Ethics, 16 (1):116-121.

CISLER, J.; BEGLE, A.; AMSTADTER, A. y ACIERNO, R. (2012). "Mistreatment and Self-Reported Emotional Symptoms: Result From the National Elder Mistreatment Study", en Journal of Elder Abuse \& Neglect, 24 (3): 216-230.

DOMÉNECH, M., e ÍNIIGUEZ, L. (2002). "La construcción social de la violencia”, en Athenea Digital, 2: 1-10, en http://atheneadigital.net/article/view/54/54 (consulta 28/7/2014).

EIRLER, R. (1997). "Human Rights and Violence: Integrating the Private and Public Spheres", en J. Turpin, J. y L.R. Kurtz (eds.) The Web of Violence. From the Interpersonal to Global. Chicago, Illinois: University of Illinois Press, pp.161-185.

ELIAS, R. (1997). "A Culture of Violent Solutions", en J. Turpin, J and L.R. Kurtz, (eds.) The Web of Violence. From the Interpersonal to Global. Chicago, Illinois: University of Illinois Press, pp.117-147.

GALTUNG, J. (2003). Paz por medios pacíficos: paz y conflicto, desarrollo y civilización. Bilbao: Bakeaz.

GALTUNG, J. (2003). Violencia cultural. Bilbao: Gernika-Gogoratuz, en: http://www.gernikagogoratu z.org/web/uploads/documentos/202892edd66aafe5c03dacf1298fd7f8938fae76.pdf(consulta 9/8/2014). GIDDENS, A. (2010). Sociología. Madrid: Alianza.

GLENDENNING, F. J. y DELCALMER, P. (1997). Mistreatment of Elderly People. Thousand Oaks, CA: SAGE Publications Ltd.

GRACIA IBÁÑEZ, J. (2011). "El maltrato familiar hacia las personas mayores. Algunas reflexiones para la delimitación de un territorio de fronteras difusas". Oñati Socio-Legal Series, 1(8): 1-26, en: http://opo.iisj.net/index.php/osls/article/view/88/140 (consulta 28/7/2014)

HEWSTONE, M. (dir.) (1994). Introducción a la Psicología Social. Barcelona: Ariel.

HOMANS, G. C. (1990). "El conductismo y después del conductismo", en A. Giddens, y J. Turner (comps.) La teoría social hoy. Madrid: Alianza Universidad, pp. 81-111.

HOMER, A.C. y GILLEARD, C. (1990). "Abuse of elderly people by their careers". British Medical Journal. 301: 1359-1362.

IBORRA, I. (2008). Maltrato de personas mayores en la familia en España. Valencia: Centro Reina Sofía. JOAS, H. (2005). Guerra y modernidad: estudios sobre la historia de la violencia en el siglo XX. Barcelona: Paidós Ibérica. 
KESSEL, H.; MARÍN, N. y MATURANA, N. (1996). "Primera Conferencia Nacional de Consenso sobre el Anciano Maltratado". Revista Española de Geriatría y Gerontología, 31: 367-372.

LAU, E. y KOSBERG, J. I. (1979). “Abuse of the Elderly by Informal Care Providers”. Aging, 297: 10-15. LEMERT, E. (1972). Human Deviance, Social Problem and Social Control. Upper Saddle River. N.J.: Prentice Hall.

MACÍAS CASTILLO, A. y LLAMAS POMBO, E. (eds.) (2008). Código derecho de la información y publicitario. Madrid: La Ley.

MOORE, B. Jr. (2001). Pureza moral y persecución en la historia. Barcelona: Paidós.

ORGANIZACIÓN MUNDIAL DE LA SALUD (2002). "El maltrato de las personas mayores", E. Krug, et al. (eds.) Informe mundial sobre la violencia y la salud. Ginebra: OMS. pp. 133-158.

ORGANIZACIÓN MUNDIAL DE LA SALUD (2002). "La violencia un problema de salud pública", en E. Krug, et al. (eds.) Informe mundial sobre la violencia y la salud. Ginebra: OMS, pp. 1-24.

ORGANIZACIÓN MUNDIAL DE LA SALUD. RED INTERNACIONAL PARA LA PREVENCIÓN DEL MALTRATO AL ANCIANO (2002). "Voces ausentes: opiniones de personas mayores sobre abuso y maltrato al mayor". Revista Española de Geriatría y Gerontología, 37(6): 319-331.

ORGANIZACIÓN MUNDIAL DE LA SALUD. RED INTERNACIONAL PARA LA PREVENCIÓN DEL MALTRATO AL ANCIANO, Y UNIVERSIDAD DE TORONTO (2002). Declaración de Toronto para la prevención global del maltrato de las personas mayores. Ginebra: OMS.

ROJAS MARCOS, L. (2008). Las semillas de la violencia. Madrid: Espasa Calpe.

ROSENWEIN, B. H. (2010). "Problems and Methods in the History of Emotions. Passions in Context". International Journal for the History and Theory of Emotions, 1: 11-32, en: http://www.passions incontext.de/index.php?id=557 (consulta 9/8/2014).

ROSENWEIN, B. H. (2002). "Worrying about Emotions in History". The American Historical Review, 107 (3): 821-845.

SANCHO, M. (comp.) (2011). Estudio de prevalencia de malos tratos a personas mayores en la Comunidad Autónoma del País Vasco. Vitoria-Gasteiz: Gobierno Vasco. Documentos de bienestar social, $\mathrm{n}^{\mathrm{o}}$ 75, en: http://www.gizartelan.ejgv.euskadi.net/contenidos/informacion/publicaciones_ss/ es_publica/adjuntos/ESTUDIO\%20DE\%20PREVALENCIA_CAST.pdf (consulta 9/8/2014).

TILLY, Ch. (2007). Violencia colectiva. Barcelona: Hacer

TURPIN, J. y KURTZ, L. (1997). "Introduction: Violence - The Micro/Macro Link", en J. Turpin, J. y L.R. Kurtz (eds.) The Web of Violence. From Interpersonal to Global. Chicago, Illinois: University of Illinois Press, pp. 1-28.

UNIÓN DEMOCRÁTICA DE PENSIONISTAS (2012). Los malos tratos económicos a personas mayores. Madrid: Simple Lógica.

UNIÓN DEMOCRÁTICA DE PENSIONISTAS (2008). Malos tratos y abusos a personas mayores. Madrid: UDP

WIEVIORKA, M. (2012). La violence. Paris: Fayard.

WORLD HEALTH ORGANIZATION (2008). A Global Response to Elder Abuse and Neglect: Building Health Care Capacity to Deal with the Problem Worldwide: Main Report. Ginebra: WHO. 


\section{Breve currículo:}

\section{José Luis Paniza Prados}

Profesor de Métodos y Técnicas de Investigación Social y de Teoría Sociológica del Departamento de Sociología de la Universidad de Granada e investigador, tanto en el ámbito del turismo y la actividad física, como en temas relacionados con la mejora de la calidad de vida de las personas mayores. Entre su más de medio centenar de publicaciones se encuentran: "Turismo y deporte: una perspectiva de análisis", "Turismo y calidad de vida en la población adulta", "Turismo Senior" o "Evolución del turismo Internacional: crisis como nueva oportunidad". También ha desarrollado tareas de investigación y consultoría para diversas empresas y para la Fundación Instituto Euroárabe de Educación y Formación.

\section{Juan Carlos Ortigosa Perochena}

Licenciado en Sociología, especialidad de psicología social, por la Universidad Complutense $\left(1^{\text {a }}\right.$ promoción de sociólogos de España) y estudios de Ciencias Físicas en la misma universidad (1967). En 1976, funda la Editorial Campo Abierto en Madrid, compilando textos como el titulado El análisis institucional. A nivel profesional, ha sido director de diversas empresas de comercio internacional y de logística fundamentalmente marítima. Máster en Problemas Sociales en la Facultad de Ciencias Políticas y Sociología por la Universidad de Granada. 